\title{
The effects of ambulatory accelerations on the stability of a magnetically suspended impeller for an implantable blood pump
}

\author{
G. Paul ${ }^{\mathrm{a}}$, M. A. Rezaienia ${ }^{\mathrm{a}}$, A. Rahideh ${ }^{\mathrm{b}}$, A. Munjiza $^{\mathrm{a}}$, T. Korakianitis ${ }^{\mathrm{c}, *}$ \\ ${ }^{a}$ School of Engineering and Materials Science, Queen Mary University of London, Mile End Road, E1 4NS, UK \\ ${ }^{b}$ School of Electrical and Computer Engineering, Shiraz University, Shiraz, Iran \\ ${ }^{c}$ Parks College of Engineering, Aviation and Technology, Saint Louis University, St. Louis, Missouri 63103, USA
}

\begin{abstract}
This paper describes the effects of ambulatory accelerations on the stability of a magnetically suspended impeller for use in implantable blood pumps. A magnetic suspension system is developed to control the radial position of a magnetic impeller using coils in the pump casing. The magnitude and periodicity of ambulatory accelerations at the torso are measured. A test rig is then designed to apply appropriate accelerations to the suspension system. Accelerations from 0 to $1 \mathrm{~g}$ are applied to the suspended impeller with ambulatory periodicity while the radial position of the impeller and power consumption of the suspension system are monitored. The test is carried out with the impeller suspended in air, water and a glycerol solution to simulate the viscosity of blood. A model is developed to investigate the effects of the radial magnetic suspension system and fluid damping during ambulatory accelerations. The suspension system reduces the average displacement of the impeller suspended in aqueous solutions within its casing to $100 \mu \mathrm{m}$ with a power consumption of below $2 \mathrm{~W}$ during higher magnitude ambulatory accelerations (RMS magnitude $0.3 \mathrm{~g}$ ). The damping effect of the fluid is also examined and it is shown that buoyancy, rather than drag, is the primary cause of the damping at the low displacement oscillations that occur during the application of ambulatory accelerations to such a suspension system.
\end{abstract}

Keywords: Magnetic bearing, third generation, VAD, ambulatory

\section{Introduction}

Congestive heart failure (CHF) affects $2 \%$ of adults in the U.S. with poor mortality rates amongst older sufferers [1]. Implantable volume displacement devices, such as intra-aortic balloon pumps [2] and skeletal muscle ventricles [3], can improve circulation and life expectancy in end stage heart failure. Implantable rotary blood pumps can also offer life-saving solutions to people with later stage $\mathrm{CHF}$ and other severe cardiovascular diseases [4]. These implantable solutions are becoming increasingly common as the demand for donor hearts greatly exceeds the supply. However, in a recent destination therapy trial for Thoratec's Heartmate II implantable blood pump, patients suffered an $11 \%$ disabling stroke rate within 2 years which was attributed to high shear stress on the blood [5]. Although numerical modelling $[6]$ and in vitro tests $[7,8]$ can be used to analyse pump performance and haemolysis, only in vivo tests allow factors like stroke rate to be gauged. Consequently improving device hemocompatibility at an early stage is vital to fabricating a low-risk implantable device.

\footnotetext{
${ }^{*}$ Corresponding author: T. Korakianitis, Parks College of Engineering, Aviation and Technology, Saint Louis University, St. Louis, Missouri 63103, USA.

Email address: Email forward for life

korakianitis@alum.mit.edu (T. Korakianitis)
}

Crucial issues are reducing shear stress [9] and residence time [10], both of which contribute to haemolysis. Improved device designs will reduce the shear stress on the blood and reduce the chance of thrombosis and stroke [11].

Second generation blood pumps are rotary, continuousflow devices, unlike their pulsatile volume displacement predecessors. Most second generation implantable blood pumps, including Heartmate II, spin on solid bearings, often made of ceramic [12] or ceramic-alumina [13], which are the main source of blood complications in these devices. More modern, third generation implantable blood pumps, including Heartmate III [14], use a non-contacting impeller as a means to prevent blood complications in medium to long term mechanical circulatory support (MCS) applications. Contact with the casing walls must be avoided to improve pump efficiency, prevent damage to the impeller and reduce shear stress on the blood which can lead to haemolysis. The pump must be able to respond to unpredictable external disturbances which will inevitably occur when a patient is ambulatory [15]. The pump must also be stabilised against gravity and against internal forces, such as axial thrust or the radial force in a centrifugal pump that occurs due to the asymmetric pressure in the volute [16].

Ideally, the impeller would be stabilised using only passive magnets, to minimise power consumption. It is gener- 
ally accepted that it is not possible to stabilise an impeller using passive magnets alone, as demonstrated analytically by the application of Earnshaw's theorem [17]. In third generation implantable blood pumps a prevalent method for fully suspending a non-contacting impeller is inserting magnets into the impeller and controlling its position with a combination of coils and magnets in the casing of the pump. Examples include the Terumo DuraHeart [18] and the WorldHeart Levacor [19]. The magnets in the casing passively suspend the magnetic impeller in one or two axes, while the coils in the casing actively suspend the impeller in the remaining free axis or axes. This allows full contactless suspension and is known as hybrid magnetic suspension [20]. Typical MCS device maglev power consumptions stated in the literature vary from $0.33 \mathrm{~W}$ (MagneVAD) to 2-4 W (Berlincor), with typical implementations requiring around $1 \mathrm{~W}$ [18]. Although hybrid magnetic suspension is the primary method of impeller control, different implantable blood pumps use various combinations of passive magnetic, active magnetic and hydrodynamic bearings to control an impeller, exemplified by Heartware's HVAD device [21] which is suspended radially by a magnetic bearing and axially by hydrodynamic forces. Hydrodynamic forces in this context refer to the local variations in blood pressure inside the casing which can be used to stabilise and suspend the impeller. One reported MCS device, Ventrassist, stabilises the impeller using only hydrodynamic forces [22]. This is achieved with a specifically customised geometry. Other customised geometries, such as spiral groove bearings, can also improve the stability of a suspended impeller [23].

Here, only magnetic bearing and fluid damping forces are examined and there is no rotation to avoid the confounding effects of lubricant pressure. This paper examines the stability during ambulatory accelerations of a magnetic cylinder actively suspended in the radial ( $\mathrm{X}$ and $\mathrm{Y}$ ) directions. A test rig is fabricated using a magnetic cylinder as the mass on the end of an inverted pendulum, which causes the mass to be unstable radially. The mass is free to move in the $\mathrm{X}$ and $\mathrm{Y}$ directions while being mechanically constrained in the $\mathrm{Z}$ direction, in a similar manner to a one-axis axial passive magnetic bearing.

The application of ambulatory accelerations is a vital tool in demonstrating that a device is suitable for an ambulatory patient [24]. In this paper accelerations were measured from the author's torso at various walking speeds for device testing. A test rig is designed to allow the application of these accelerations to the system and investigate the stability of the impeller in air and in fluid. The aim of the tests in this paper is to investigate the behaviour of the radial magnetic suspension system in different fluids by examining impeller stability and power consumption during ambulatory accelerations. The development of a mathematical model to predict the transient behaviour of an impeller allows better understanding of the mechanism behind its damping in fluid and the prediction of its behaviour with blood as the working fluid. Air was used as a working fluid to allow verification of the dynamic model without damping. This ensures the model is correctly describing the magnetic bearing when no damping is present, and gives us greater confidence in modelling the fluid damping. To our knowledge, an analysis of the response of this type of submerged bearing system to ambulatory accelerations has not been previously reported.

\section{Experimental methods}

The bearing system is composed of a magnetic cylinder, representing and hereafter referred to as an impeller, as the mass of an inverted pendulum in a cylindrical casing, shown in the cross-section Figure 1. This figure also shows the configuration of magnets and coils to control movement in a single axis. The casing and pendulum are fabricated using 3D printed ABS and machined clear acrylic components.

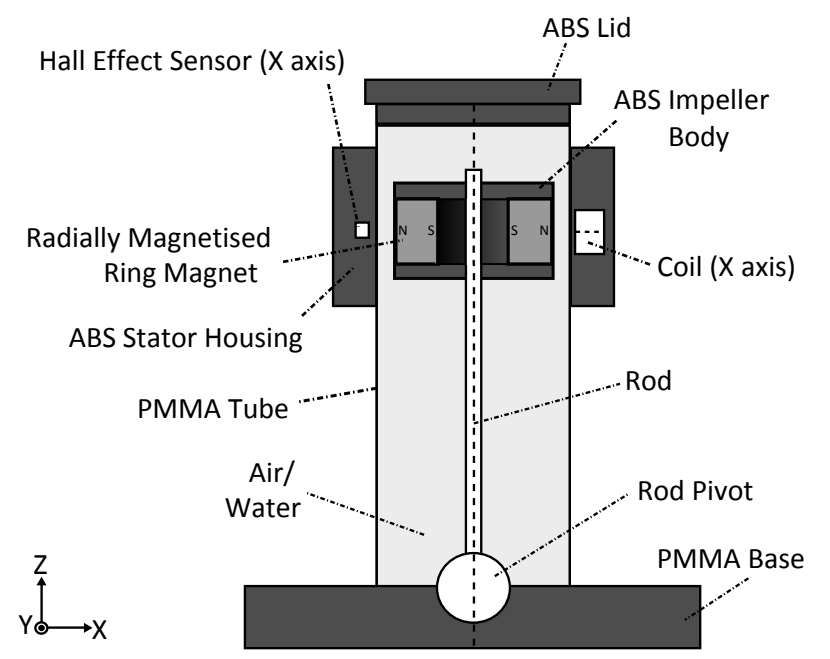

Figure 1: Cross-sectional view of the inverted pendulum setup for testing the radial magnetic bearing.

The magnetic impeller is axially constrained on the rod and radially stabilised by an active magnetic bearing system. The rod is fixed in a frictionless pivot at the bottom of the casing so that the weight of the impeller pushes it away from the centre, providing a symmetric radial instability. The pendulum has length, l, $90 \mathrm{~mm}$ from the pivot to the centre of mass of the impeller, and a rod diameter of $3 \mathrm{~mm}$. The impeller has a diameter of $29 \mathrm{~mm}$ and mass, $\mathrm{m}$, of $65 \mathrm{~g}$, which is at the low end of devices reported in the literature. Although impeller weights are rarely disclosed, full device weights ranging from 145g (HeartWare HVAD) to $540 \mathrm{~g}$ (Terumo DuraHeart) are reported [11]. The impeller contains a radially magnetised Nd-Fe-B ring magnet, grade N35, supplied by Magnet Sales. The casing has an inner diameter of $30.8 \mathrm{~mm}$, an outer diameter of $31.8 \mathrm{~mm}$ and a length of $120 \mathrm{~mm}$.

Radially magnetised ring magnets are appropriate for radial active stabilisation and position sensing. The use of a ring magnet allows the impeller's $\mathrm{X}$ and $\mathrm{Y}$ position 
to be sensed accurately with a pair of Hall effect sensors. The impeller's radial position can be controlled using a single ring magnet in the impeller and one coil per axis. Optimisation of the dimensions of coils and magnets used in this work has not been performed. Two separate electromagnetic control systems are responsible for controlling the $\mathrm{X}$ and $\mathrm{Y}$ position of the impeller. Each consists of a single Hall effect sensor and a single coil. The placement of the coils, ring magnet and Hall effect sensors is shown in Figure 2. A centrifugal impeller into which such a system could be inserted is shown in Figure 3. This method of suspension has previously been described by Hijikata et al [25] and Matsamura et al [26] among others.

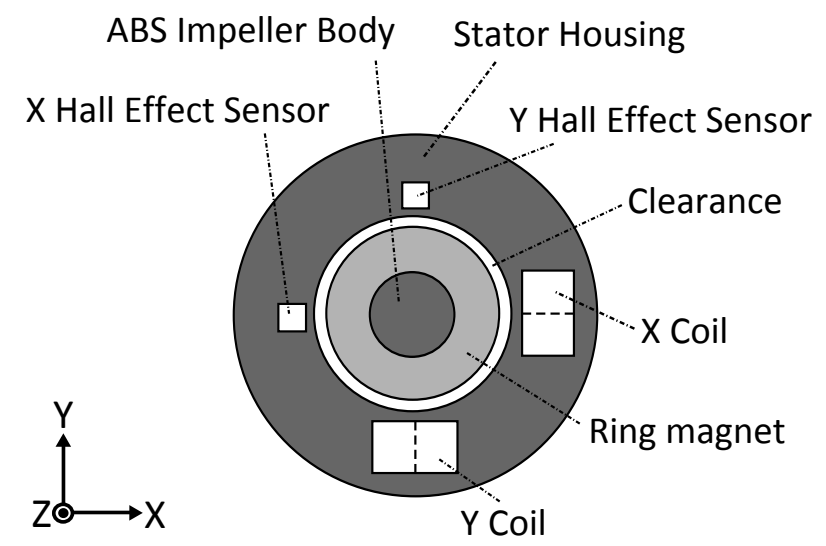

Figure 2: Cross-section of the radial active suspension system in the $\mathrm{X}-\mathrm{Y}$ plane.

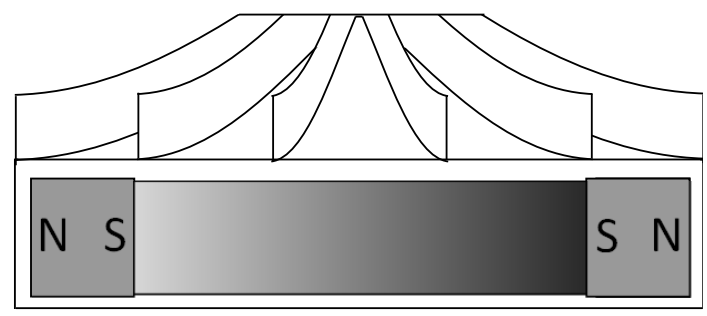

Figure 3: Implementation of this suspension system in a typical centrifugal impeller used in MCS.

The ring magnet has an outer diameter of $29 \mathrm{~mm}$, an inner diameter of $18 \mathrm{~mm}$ and a thickness of $5 \mathrm{~mm}$. The coils have an outer diameter of $12 \mathrm{~mm}$, an inner diameter of $3 \mathrm{~mm}$ and a thickness of $6 \mathrm{~mm}$, and are composed of 220 turns of enamelled copper wire. When the ring magnet is in the centre, the clearance between the ring magnets and the inner wall of the casing is $0.9 \mathrm{~mm}$ and the spacing between the magnets and Hall effect sensors is $6.4 \mathrm{~mm}$. The spacing between the coils and the ring magnet can vary from 0.5 to $2.3 \mathrm{~mm}$ and is $1.4 \mathrm{~mm}$ in the central position.

An analog electronics implementation is used in this system. Two proportional-derivative (PD) control systems are used to control the radial displacement. The full analog control system is composed of Hall effect sensors, sen- sor amplification, PD controllers, PWM modulation and power transistors. A diagram of the control system for a single axis is given in Figure 4.

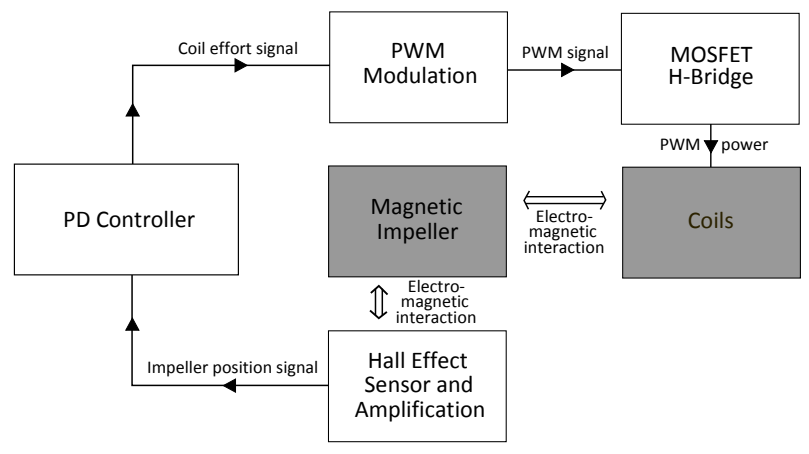

Figure 4: A block diagram of the full analog control system for a single axis. Blocks representing electromechanical components are shaded.

A PD controller was selected for this experiment because it provides the capability to control the impeller during movement. A PID controller would not adversely affect performance but for reduced complexity and focus on the dynamic scenario a PD controller was used. The absence of an integrator (I) element in the controller creates a non zero steady state error but the authors believe it will not significantly affect the dynamic scenario examined here. The bearing was tuned by increasing the proportional gain until the maximum current $(2.5 \mathrm{~A})$ was reached approximately when the impeller was touching the casing (0.9 $\mathrm{mm}$ displacement). The derivative gain was then increased gradually until the impulse response showed no oscillation. This manual tuning method does not provide optimal performance but is sufficient for this investigation. The final proportional gain was $3.1 \mathrm{~A} / \mathrm{mm}$ and the final derivative gain was 0.05 A.s $/ \mathrm{mm}$.

When there is no applied acceleration in the radial direction, the impeller is held in the middle of its casing with minimal pulsing of the $\mathrm{X}$ or the $\mathrm{Y}$ coils. The power through the coils is less than $50 \mathrm{~mW}$ in air and less than $20 \mathrm{~mW}$ in liquids. The main power consumption in this situation is that of the control circuit and the Hall effect sensors, roughly $140 \mathrm{~mW}$. This power consumption should be compared to the typical power consumption of a mechanical circulatory support device, which typically ranges from 10-20 W. The Heartmate III is reported to achieve $7 \mathrm{~L} / \mathrm{min}$ with $135 \mathrm{~mm} . \mathrm{Hg}$ pressure head with a fully suspended impeller and a power consumption below $10 \mathrm{~W}$ [27]. In the tests reported here the instantaneous power consumption through the radial suspension coils is limited at $18.75 \mathrm{~W}$, although clearly this would not be sustainable in a ventricular assist device. The excitation voltage of the coils is $7.5 \mathrm{~V}$ and the maximum current is $2.5 \mathrm{~A}$.

The radial bearing is tested in an ambulatory scenario to give an indication of bearing performance during everyday activities. Ambulatory accelerations were measured 
from the author on a treadmill using an ADXL335 accelerometer with the bandwidth set to $50 \mathrm{~Hz}$ attached to the chest near the location of the heart. An oscillating cart is then used to simulate the magnitude and periodicity of the measured ambulatory accelerations with the bearing test rig. The cart, which has the radial magnetic suspension system and an accelerometer attached, moves in the $\mathrm{X}-\mathrm{Y}$ plane only. It provides single-axis accelerations to a cart with an oscillation frequency which can be adjusted to the desired frequency by adding weights. The inverted pendulum is oriented so that both $\mathrm{X}$ and $\mathrm{Y}$ axis control systems are at 45 degrees to the direction of acceleration. A top-down diagram of the test rig is shown in Figure 5.

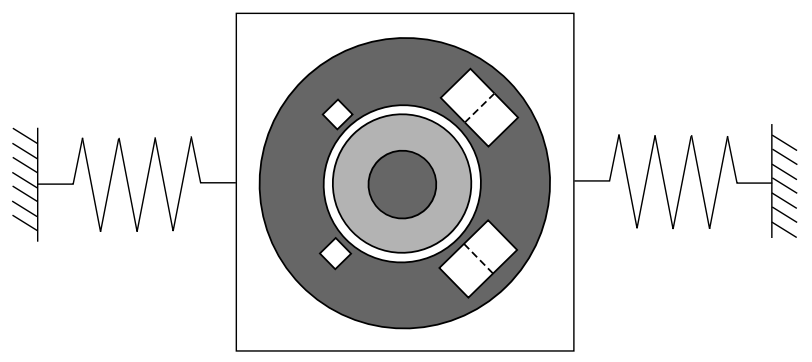

Figure 5: Test rig to apply one dimensional accelerations to the inverted pendulum in the $\mathrm{X}-\mathrm{Y}$ plane.

By releasing the cart from various displacements, a range of different accelerations can be tested. A data acquisition system (USB-6008) is used to monitor the amplified Hall effect sensor readings and measure the impeller displacement in the $\mathrm{X}$ and $\mathrm{Y}$ axes, to monitor the acceleration of the cart and to monitor the power consumption of the system. The USB-6008 data acquisition system samples at $1000 \mathrm{~Hz}$ with a 10-bit resolution. This test rig is used to examine the behaviour of the magnetic bearing system in air, water and a $40 \%$ glycerol solution which has a density of $1100 \mathrm{~kg} / \mathrm{m}^{3}$ and a viscosity of $3.72 \mathrm{mPa} . \mathrm{s}$ at $20{ }^{\circ} \mathrm{C}$ [28]. The $40 \%$ glycerol solution is used in MCS research to simulate the behaviour of blood, which has a density of $1060 \mathrm{~kg} / \mathrm{m}^{3}$ and a viscosity of $3.4 \mathrm{mPa}$.s. The temperature when this experiment was performed was 22 ${ }^{\circ} \mathrm{C}$. The acceleration of the cart during a single trial, during which it is displaced and then released, is shown in Figure 6.

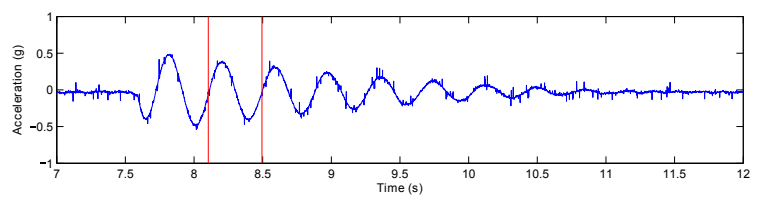

Figure 6: Harmonic response of the cart to being displaced then released. Red lines show the second full oscillation.

In order to extract data from these experiments, the second full oscillation of each trial was defined as the sampling region, shown with vertical lines in Figure 6. From this region, the RMS acceleration, RMS displacement of the impeller and mean current consumption of the system are extracted. The second full oscillation was chosen as it would allow high magnitudes to be observed while giving the system time to settle into its oscillation.

\section{Analytical Modelling}

The forces on the impeller during these accelerations were calculated using a model implemented in MATLAB. This model was implemented primarily to find what factors affected the performance of the bearing in air and in water. In the model, the acceleration of the cart is applied as a fixed amplitude sin wave, and from this the cart speed and displacement are known. The bearing applies a force to the impeller to keep it central in its casing and prevent wall collisions as the cart moves. The vectors $\mathrm{x}$ and $\mathrm{F}$ represent displacement and force in the same direction across the axis of applied acceleration. The instability of the inverted pendulum is approximated as $F_{\text {pendulum }}=m \cdot g \cdot \sin (\theta)=m \cdot g \cdot x / l$, where $\mathrm{m}$ is the mass, $\mathrm{l}$ is the shaft length, $\mathrm{x}$ is the displacement relative to the casing and $\theta$ is the angle between the pendulum shaft and the $\mathrm{Z}$ axis.

The magnetic characteristics of one axis of the bearing system are calculated using Ansys Maxwell to give the force per applied current and the magnetic field at the Hall sensors across the full $1.8 \mathrm{~mm}$ range. This program uses a $\mathrm{T}-\Omega$ solver to solve Maxwell's equations for magnetic fields and Eddy currents within the domain when a current is applied through a conductor. A magnetostatic simulation with a parametric sweep of displacement $0.1125 \mathrm{~mm}$ was used. The simulation had 100,000 tetrahedral elements and solved when the energy error was below $0.1 \%$. This gave the results shown in Figures 7-9.

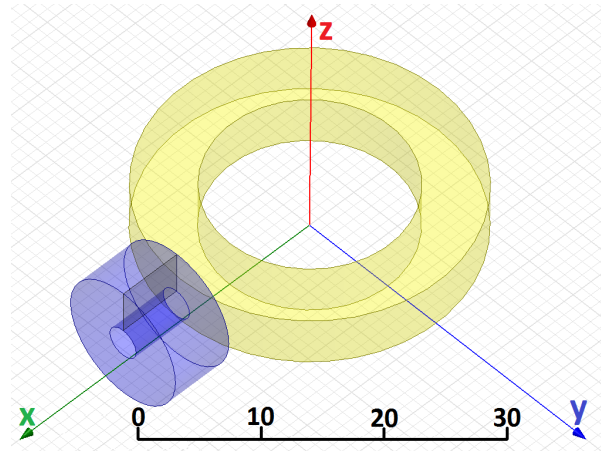

Figure 7: The simulation model with scale in $\mathrm{mm}$.

The force per amp of current through the coil varies depending on magnet position and will be modelled by the formula $F=0.2+0.57 x \mathrm{~N} / \mathrm{A}$ as shown in Figure 8. The in-circuit gains for proportional and derivative elements are $3.1 \mathrm{~A} / \mathrm{mm}$ and $0.05 \mathrm{~A} . \mathrm{s} / \mathrm{mm}$. Together these calculations give the theoretical bearing characteristics which are proportional stiffness, $\mathrm{k}_{p}$, of $620 \mathrm{~N} / \mathrm{m}$ and a derivative 


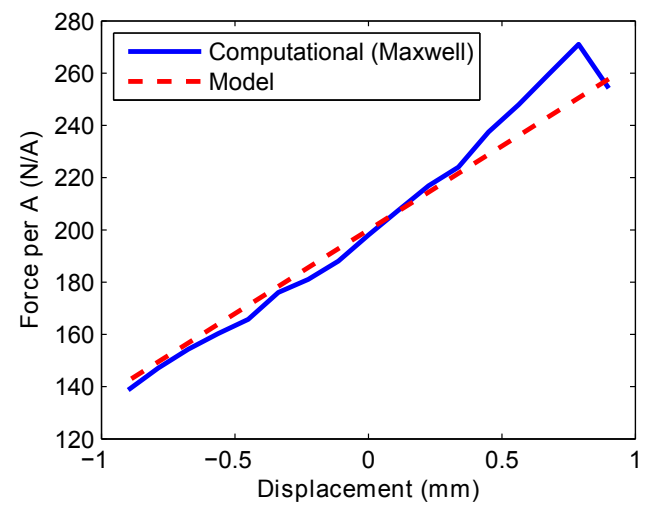

Figure 8: The computational and modelled force per amp.

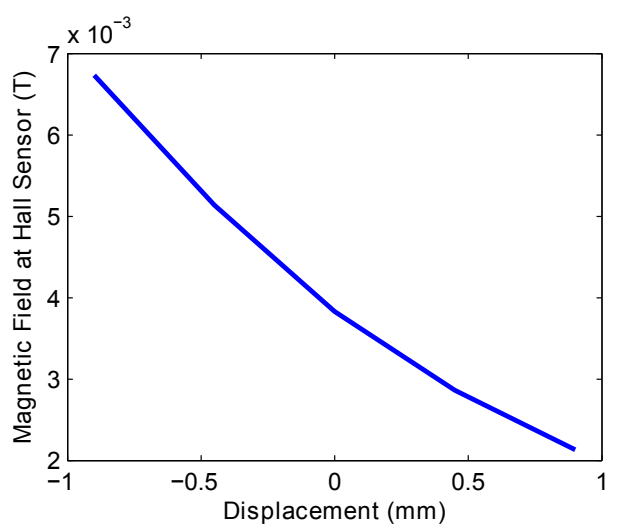

Figure 9: The magnetic field at the Hall sensor.

stiffness, $\mathrm{k}_{d}$, of $10 \mathrm{~N} . \mathrm{s} / \mathrm{m}$ when the impeller is radially centralised. The airgap-force characteristic shown in Figure 8 is used in the model. In the model, the bearing forces in the direction of the applied acceleration are simulated on the impeller. The forces from $\mathrm{X}$ and $\mathrm{Y}$ coils are shown in Figure 10.

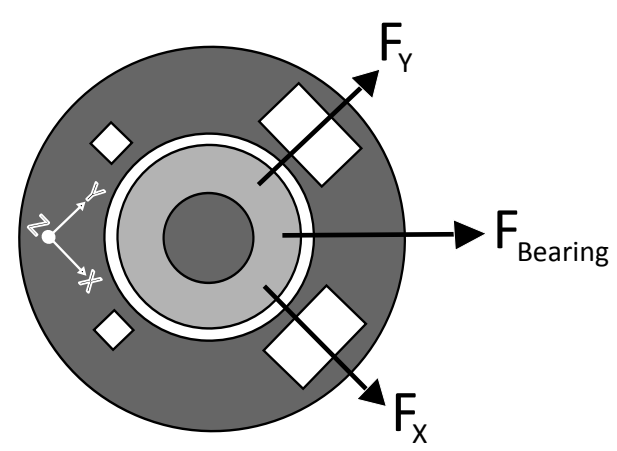

Figure 10: The orientation of the test rig during the applied accelerations.

Since the coils and Hall sensors are all at $45^{\circ}$ angles to the impeller in the test configuration, the sensed displacement and speed for $\mathrm{X}$ and $\mathrm{Y}$ axes are $x / \sqrt{2}$ and $\dot{x} / \sqrt{2}$ respectively, where $\dot{x}$ is the relative velocity of the impeller. The resultant bearing force is given in equation 1 .

$$
\begin{aligned}
F_{\text {Bearing }}=\frac{F_{X}+F_{Y}}{\sqrt{2}}=\frac{2}{\sqrt{2}}\left(-k_{p} \cdot \frac{x}{\sqrt{2}}-k_{d} \cdot \frac{\dot{x}}{\sqrt{2}}\right) \\
=-\left(k_{p} \cdot x+k_{d} \cdot \dot{x}\right)
\end{aligned}
$$

When submerged, additional forces due to buoyancy and viscosity must be included in the model. Buoyancy is usually considered to act upwards against gravity, but in this case the radial accelerations cause an additional radial buoyancy force. This force has magnitude $\rho . V$. $a$ where $\rho$ is the density of water, $\mathrm{V}$ is the volume of the impeller and a is the acceleration of the cart. a should not be confused with $\ddot{x}$, which is the acceleration of the impeller relative to its casing. Viscous forces were also calculated using Van Hyde's formula for the drag coefficient $\left(C_{d}\right)$ of a cylinder at low Reynolds numbers [29], given in equation 2. The total fluid damping forces are given in equation 3 , where $\mathrm{A}$ is the projected surface area of the pump.

$$
\begin{gathered}
C_{d}=\frac{4 \pi}{R e}\left(\Delta_{1}-0.87 \Delta_{1}^{3}\right), \Delta_{1}=\frac{1}{\log \left(\frac{4}{R e}\right)-\gamma-\frac{1}{2}} \\
F_{\text {Damping }}=\frac{1}{2} \cdot \rho \cdot C_{d} \cdot A \cdot \dot{x}^{2}+\rho \cdot V \cdot a
\end{gathered}
$$

The calculated Reynolds numbers are 334 in water and 80 in the glycerol solution. This is significantly lower than Reynolds numbers in blood pumps which are typically around 33000 [30]. The total modelled instantaneous force on the impeller in air and liquid is given in equation 4. This is implemented in the MATLAB dynamic model to allow a prediction of the displacement of the impeller relative to its casing at different applied accelerations.

$$
\begin{gathered}
F_{\text {Impeller }}=m \cdot \ddot{x}=F_{\text {Pendulum }}+F_{\text {Bearing }}+F_{\text {Damping }} \\
\quad=m \cdot g \cdot \frac{x}{l}-k_{p} \cdot x-k_{d} \cdot \dot{x}-\frac{1}{2} \cdot \rho \cdot C_{d} \cdot A \cdot \dot{x}^{2}+\rho \cdot V \cdot a
\end{gathered}
$$

\section{Results}

Accelerations in the front-to-back $(\mathrm{X})$, side-to-side $(\mathrm{Y})$ and up-to-down $(\mathrm{Z})$ directions were measured. Ambulatory accelerations were collected during walking at 1.25 and $1.75 \mathrm{~Hz}$ pace frequencies. The reasoning behind these frequencies was that $1.25 \mathrm{~Hz}$, a slow walk, could represent the pace frequency of an ill patient while $1.75 \mathrm{~Hz}$, a brisk walk, could represent the pace frequency of a fully rehabilitated patient. Ambulatory accelerations at 1.25 and $1.75 \mathrm{~Hz}$ walking frequency are shown below in Figures 11 and 12 .

The RMS accelerations for the $\mathrm{X}, \mathrm{Y}$ and $\mathrm{Z}$ directions were $0.099 \mathrm{~g}, 0.126 \mathrm{~g}$ and $0.145 \mathrm{~g}$ at $1.25 \mathrm{~Hz}$ and $0.152 \mathrm{~g}$, $0.148 \mathrm{~g}$ and $0.310 \mathrm{~g}$ at $1.75 \mathrm{~Hz}$. FFT analysis showed a spike at the fundamental walking frequency and other high amplitudes between 2 and $6 \mathrm{~Hz}$. The oscillation frequency 

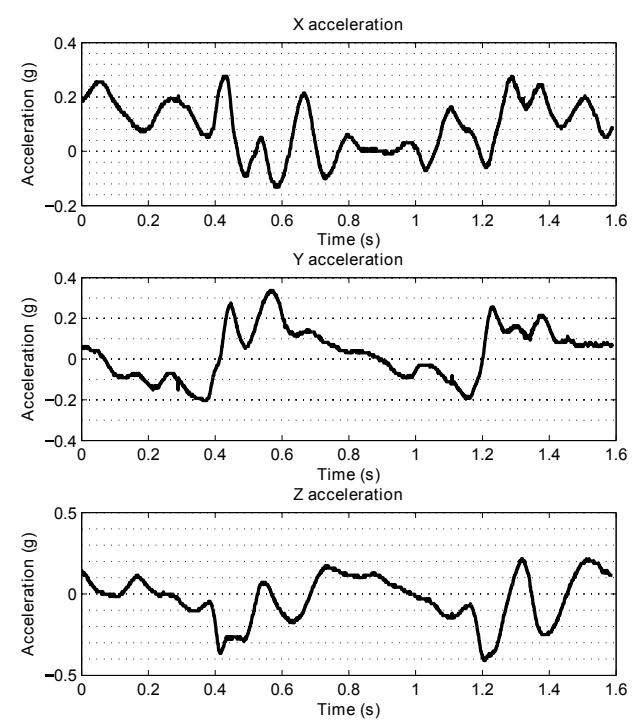

Figure 11: Ambulatory accelerations at pace frequencies of $1.25 \mathrm{~Hz}$.
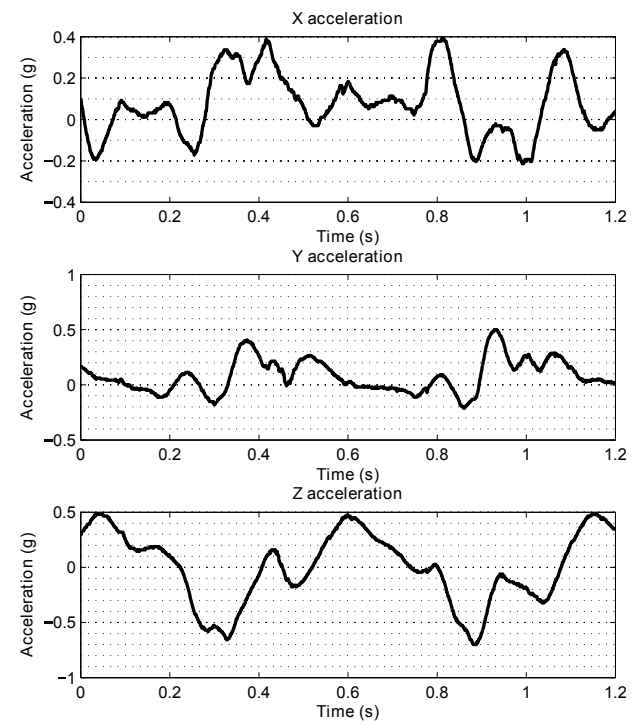

Figure 12: Ambulatory accelerations at pace frequencies of $1.75 \mathrm{~Hz}$.

of the cart was set at $2.5 \mathrm{~Hz}$. The magnetic bearing system was tested under several acceleration magnitudes with the impeller suspended in air, water and the glycerol solution. A range of different RMS accelerations of 0.05 to $0.7 \mathrm{~g}$ were tested. The trials in air were stopped at RMS accelerations of $0.45 \mathrm{~g}$ because the maximum power consumption was reached during peaks of acceleration. More than 20 trials were carried out with each working fluid. Figure 13 shows the recorded data from the sampling region (second full oscillation) of a single trial in air, with RMS acceleration $0.2 \mathrm{~m} / \mathrm{s}^{2}$.

For each trial the RMS cart acceleration, mean power consumption and RMS displacement are calculated from the sampling region giving the results shown in Figure 14
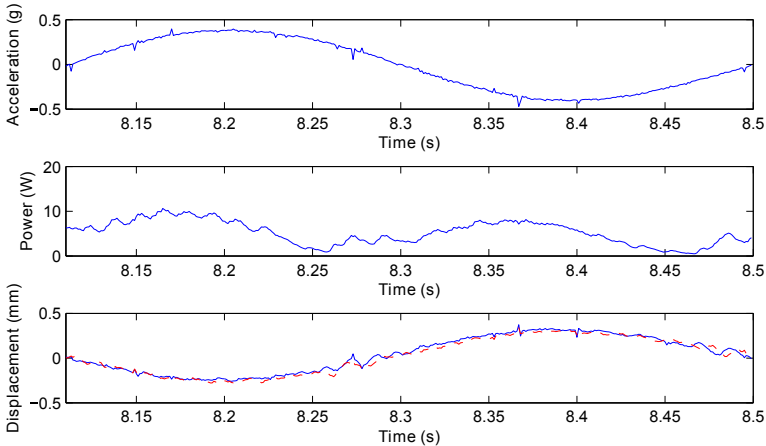

Figure 13: Acceleration and current consumption of the magnetic bearing system and displacement of the impeller during the sampling region of the trial in air. Displacement is shown in solid blue for the $\mathrm{X}$ axis and dashed red for the $\mathrm{Y}$ axis.

and Figure 15. The RMS displacement calculated by the analytical model is also shown. The stated current consumption is the sum of that of the $\mathrm{X}$ and $\mathrm{Y}$ coils, while the stated displacement is in the direction of applied acceleration (see Figure 10). All data points are shown.

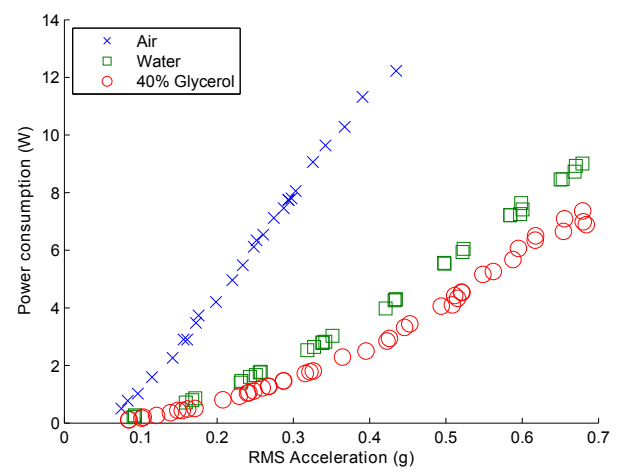

Figure 14: Mean power consumption against RMS acceleration.

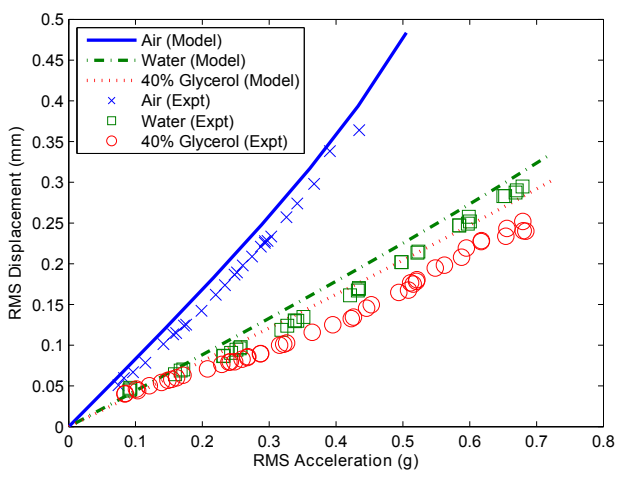

Figure 15: Experimental and model results for the RMS displacement of the impeller at various RMS accelerations.

There is a clear reduction in the displacement of the impeller and the power consumption of the system when the impeller is submerged. At RMS accelerations of $0.45 \mathrm{~g}$ the 
maximum instantaneous power was reached in air, however wall collisions had still not occurred. In water RMS acceleration up to $0.7 \mathrm{~g}$ were applied with RMS impeller displacements remaining below $0.3 \mathrm{~mm}$, although the average power consumption at this acceleration level was around $8 \mathrm{~W}$. At all accelerations of the magnitude measured in the ambulatory testing, power consumption is below $2 \mathrm{~W}$ and the RMS displacement is below $100 \mu \mathrm{m}$. Accelerations below $0.05 \mathrm{~g}$ could not be tested experimentally because the cart did not oscillate in a repeatable and comparable fashion as required. The graph suggests that the power consumption is very low at accelerations below $0.05 \mathrm{~g}$ and the bearing system appears to consume the same level of power as when holding the impeller in the centre.

The experimental results show that there is only slightly more damping in the glycerol solution than in water. As viscous damping, $\mu$, is proportional to the dynamic viscosity, we would expect damping in the glycerol solution ( $\mu=0.0036$ Pa.s $)$ to be far greater than in water $(\mu=$ $0.00089 \mathrm{~Pa} . \mathrm{s})$. Consequently the primary effect is more likely to be due to the change in density from the glycerol solution $\left(\rho=1.1 \mathrm{~g} / \mathrm{cm}^{3}\right)$ to water $\left(\rho=1 \mathrm{~g} / \mathrm{cm}^{3}\right)$. The model gave a reasonably accurate fit for the displacement of the impeller in the three fluids. For all three the experimental displacement is slightly lower than the analytical model predicts, suggesting that the efficacy of magnet-coil interaction may have been underestimated. The model also offers further evidence that buoyancy has a greater effect than viscous damping in this system. The viscous force term in the model was very low and it is thought that the majority of damping effects were due to buoyancy as opposed to viscosity. Radial buoyancy forces were at least $10^{6}$ larger than viscous damping forces, although it is possible that the formula used for viscosity was not appropriate for a cylinder enclosed in a cylindrical casing with small clearances. The higher Reynolds number encountered with a spinning impeller will also increase the magnitude of viscous damping.

Since the distance between the ring magnet and coils is constantly changing, the strength of the force between the coil and magnet for a given excitation is also changing. This can be observed in the results in the profiles of current consumption and displacement. The displacement in the negative $\mathrm{X}$ and $\mathrm{Y}$ directions, where the ring magnet is further from the coils, is greater than the displacement in the positive, especially at large amplitudes. As a consequence, the current consumption is also greater. This can be observed in all recordings from this bearing system and is shown in the behaviour of the system in water in Figure 16.

\section{Conclusions}

The system successfully reduced radial movements of a magnetically suspended impeller during ambulatory accelerations that would be applied to an implanted MCS system. The displacement of such an impeller suspended
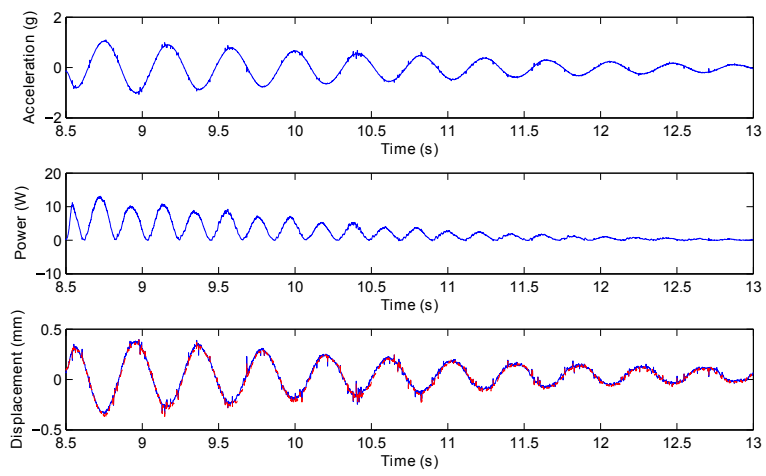

Figure 16: Response of the system to sinusoidal accelerations with the impeller in water. Displacement is shown in blue for the $\mathrm{X}$ axis and red for the $\mathrm{Y}$ axis.

in water or blood within its casing is limited to $100 \mu \mathrm{m}$ with a power consumption of $2 \mathrm{~W}$ during ambulatory accelerations with RMS magnitude equal to $\mathrm{Z}$ acceleration at a pace frequency of $1.75 \mathrm{~Hz}$, which is the largest acceleration profile measured in this paper. Larger accelerations can be experienced during a fall, climbing stairs and other situations but this provides a demonstration of the bearing performance during everyday activity.

Analysis of the results and analytical modelling showed that the main cause of the damping in this system was a buoyancy effect due to the weight of the displaced fluid under acceleration, rather than viscous drag forces. This is largely because applied accelerations in this scenario are high (over $10 \mathrm{~m} / \mathrm{s}^{2}$ ) while impeller velocity through fluid is very low (under $0.02 \mathrm{~m} / \mathrm{s}$ ). This has implications for the design of blood pumps, as the projected surface area has minimal effect on damping, while the density of the impeller is crucial. Typically titanium $\left(4.5 \mathrm{~g} / \mathrm{cm}^{3}\right)$ and rareearth magnets $\left(7.5 \mathrm{~g} / \mathrm{cm}^{3}\right)$ are used for impeller construction; minimising the amount required and using lighter materials wherever possible in the impeller will improve the stability of the impeller against external accelerations. A neutrally buoyant impeller, with the same density as the working fluid, would not move within its casing with externally applied accelerations. Consequently, viscous damping, which is proportional to velocity squared, would be very low as the impeller would not be moving through the fluid.

This paper described a novel method for magnetic bearing system performance testing in ambulatory scenarios using a spring-mass-damper oscillator. A linear motor was considered to implement more physiological accelerations, but for this experiment a mass-spring-damper oscillator was selected on the basis that its response would be sufficient to examine the behaviour of the system as well as simpler to model. The power consumption of the system during ambulatory accelerations is within acceptable limits, less than $20 \%$ of the typical power consumption of a state of the art implantable centrifugal blood pump, roughly $10 \mathrm{~W}$. This could be further reduced with optimisation 
of the coils and ring magnet to improve the efficiency of the electromagnetic interaction. The controller could be changed to a PID or MPC controller, which would improve response time and reduce displacement in the casing. The volume occupied by the electromagnetic components (coils, magnet and Hall effect sensors) is $5.8 \mathrm{~cm}^{3}$, making this system appropriate for use in implantable blood pumps where volume is a critical issue. In terms of the impeller described in this paper, the volume ratio of the impeller to magnet is $7: 1$. Reducing the size, power consumption and hemolysis are all critical issues for the continued development of mechanical circulatory support devices. Future research will examine the integration of this magnetic suspension system with the hydrodynamic forces in a spinning impeller.

\section{Acknowledgments}

This report is independent research funded by the National Institute for Health Research [i4i, Turbocardia, IILB-1111-20007]. Principal Investigator for the grant is Prof. T. Korakianitis. The views expressed in this publication are those of the authors and not necessarily those of the NHS, the National Institute for Health Research or the Department of Health.

\section{References}

[1] D. D. Schocken, M. I. Arrieta, P. E. Leaverton, and E. A. Ross. Prevalence and mortality-rate of congnsive-heart-failure in the united-states. Journal of The American College of Cardiology, 20(2):301-6, Aug. 1992.

[2] I. Ramnarine, M. Capoccia, Z. Ashley, H. Sutherland, M. Russold, N. Summerfield, S. Salmons, and J. Jarvis. Comparison between the power output of a skeletal muscle ventricle and the left ventricle in the same circulation. Artificial Organs, 28:766$67,2004$.

[3] G.A. Thomas, R.L. Hammond, K. Greer, H. Lu, J.C. Jarvis, A.R. Shortland, D.M. Pullan, S. Salmons, and L.W. Stephenson. Functional assessment of skeletal muscle ventricles after pumping for up to four years in circulation. Annals Thoracic Surgery, 70:1281-90, 2000.

[4] D. Bluestein, S. Einav, and M.J. Slepian. Device thrombogenicity emulation: a novel methodology for optimizing the thromboresistance of cardiovascular devices. Journal of Biomechanics, 361(23):2241-51, 2013.

[5] M.S. Slaughter, J.G. Rogers, C.A. Milano, S.D. Russell, J.V. Conte, D. Feldman, B. Sun, A.J. Tatooles, R.M. Delgado, J.W. Long, T.C. Wozniak, W. Ghumman, D.J. Farrar, and O.H. Frazier. Advanced heart failure treated with continuous-flow left ventricular assist device. New England Journal of Medicine, 361(23):2241-51, 2009.

[6] T. Korakianitis and Y. Shi. Numerical simulation of cardiovascular dynamics with healthy and diseased heart valves. Journal of Biomechanics, 39:1964-82, 2006.

[7] M. A. Rezaienia, A. Rahideh, M. T. Rothman, S. A. Sell, K. Mitchell, and T. Korakianitis. In vitro comparison of two different mechanical circulatory support devices installed in series and in parallel. Artificial Organs, 38:800-809, 2014.

[8] M. A. Rezaienia, A. Rahideh, B. A. Hamedani, D. E.M Bosak, Silvia. Zustiac, and T. Korakianitis. Numerical and in vitro investigation of a novel mechanical circulatory support device installed in the descending aorta. Artificial Or, Article first published online, 2015.
[9] X. Song, A. Throckmorton, H. Wood, J. Antaki, and D. Olsen. Quantitative evaluation of blood damage in a centrifugal vad by computational fluid dynamics. Journal of Fluids Engineering, 126(3):410-8, 2004.

[10] A.P. Shortland, J.C. Jarvis, and S. Salmons. Haemodynamic considerations in the design of a skeletal muscle ventricle. Medical $\&$ Biological Engineering \& Computing, 41(5):52935, 2003.

[11] D. Timms. A review of clinical ventricular assist devices. Medical engineering and physics, 33(9):1041-7, 2011.

[12] O.H. Frazier, T.J. Myers, I.D. Gregoric, T. Khan, R. Delgado, M. Croitoru, K. Miller, R. Jarvik, and S. Westaby. Initial clinical experience with the jarvik 2000 implantable axial-flow left ventricular assist system. Circulation, 105:2855-60, 2002.

[13] B. P. Griffith, R. L. Kormos, H. S. Borovetz, K. Litwak, J. F. Antaki, V. L. Poirier, and K. C. Butler. Heartmate ii left ventricular assist system: From concept to first clinical use. Annals of Thoracic Surgery, 71(3, S):S116-S120, Mar. 2001.

[14] K. Bourque, D. B. Gernes, H. M. Loree, J. S. Richardson, V. L. Poirier, N. Barletta, A. Fleischli, G. Foiera, T. M. Gempp, R. Schoeb, K. N. Litwak, T. Akimoto, M. J. Watach, and P. Litwak. Heartmate iii: Pump design for a centrifugal lvad with a magnetically levitated rotor. ASAIO Journal, 47(4):401-405, Jul.-Aug. 2001.

[15] J. Asama, T. Shinshi, H. Hoshi, S. Takatani, and A. Shimokohbe. A compact highly efficient and low hemolytic centrifugal blood pump with a magnetically levitated impeller. Artificial Organs, 30(3):160-7, 2006.

[16] O. Demir, E. Biyikli, I. Lazoglu, and S. Kucukaksu. Design of a centrifugal blood pump: Heart turcica centrifugal. Artificial Organs, 35(7):720-5, 2011.

[17] R. Bassani. Earnshaw (18051888) and passive magnetic levitation. Meccanica, 41(4):375-89, 2006.

[18] H. Hoshi, T. Shinshi, and S. Takatani. Third-generation blood pumps with mechanical noncontact magnetic bearings. Artificial Organs, 30(5):324-338, 2006.

[19] G.B. Bearnson, D.B. Olsen, P.S. Khanwilkar, J.W. Long, M. Sinnott, A. Kumar, P.E. Allaire, M. Baloh, and J. Decker. Implantable centrifugal pump with hybrid magnetic bearings. ASAIO Journal, 44:733-6, 1998.

[20] P. Khanwilkar, D. Olsen, G. Bearnson, P.E. Allaire, E. Maslen, R. Flack, and J. Long. Using hybrid magnetic bearings to completely suspend the impeller of a ventricular assist device. $\mathrm{Ar}$ tificial Organs, 20(5):1525-94, 1996.

[21] R. Wampler, D. Lancisi, V. Indravudh, R. Gauthier, and R. Fine. A sealess centrifugal blood pump with passive magnetic and hydrodynamic bearings. Artificial Organs, 23:7804, 1999.

[22] P.A. Watterson, J.C. Woodard, V.S. Ramsden, and J.A. Reizes. Ventrassist hydrodynamically suspended, open, centrifugal blood pump. Artificial Organs, 24(6):475-7, 2000.

[23] Y. Nakamura, H. Tsukamoto, K. Miyazaki, and Y. Qian. Experimental study of dynamic characteristics of a centrifugal blood pump with a conical spiral groove bearing for a ventricular assist device. In Proceedings of 5th Joint Fluids Engineering Conference, San Diego, California, 2007. ASME.

[24] G. Paul, R. Torah, S. Beeby, and J. Tudor. Novel active electrodes for ecg monitoring on woven textiles fabricated by screen and stencil printing. Sensors and Actuators: A, 221:60-6, 2015.

[25] W. Hijikata, T. Shinshi, and S. Takatani. Disposable magneticsuspension centrifugal pump, 2011.

[26] F. Matsamura and T. Yoshimoto. System modelling and control design of a horizontal-shaft magnetic-bearing system. IEEE Transactions on Magnetics, 22(3):196-203, 1986.

[27] H.M. Loree, K. Bourque, D.B. Gernes, J.S. Richardson, V.L. Poirier, N. Barletta, A. Fleischli, G. Foiera, T.M. Gempp, and R. Schoeb. The heartmate iii: design and in vivo studies of a maglev centrifugal left ventricular assist device. Artificial Organs, 25(5):386-91, 2001.

[28] J.B. Segur and H.E. Oberstar. Viscosity of glycerol and its aqueous solutions. Industrial and Engineering Chemistry, 43(9):2117-20, 1951. 
[29] M. Van Dyke. Perturbation methods in fluid mechanics. Parabolic, Stanford, 2 edition, 1975.

[30] D. Timms. Design, development and evaluation of centrifugal ventricular assist devices. PhD thesis, Queensland University of Technology Brisbane, 2005. 